Original article

\title{
Impact of cigarettes' filter length and diameter on cigarette smoke emissions
}

\author{
Jalal Poorolajal ${ }^{\mathrm{a}, \mathrm{b}}$, Mohammad Javad Assari ${ }^{\mathrm{c}}$, Younes Mohammadi ${ }^{\mathrm{d}}$, Fatemeh Gohari-Ensaf ${ }^{\mathrm{a}, *}$ \\ ${ }^{a}$ Department of Epidemiology, School of Public Health, Hamadan University of Medical Sciences, Hamadan, Iran \\ ${ }^{\mathrm{b}}$ Research Center for Health Sciences, Hamadan University of Medical Sciences, Hamadan, Iran \\ ${ }^{\mathrm{c}}$ Department of Occupational Health, School of Public Health, Hamadan University of Medical Sciences, Hamadan, Iran \\ ${ }^{\mathrm{d}}$ Modeling of Noncommunicable Diseases Research Center, Hamadan University of Medical Sciences, Hamadan, Iran
}

\section{A R T I C L E I N F O}

\section{Keywords:}

Smoking

Tobacco products

Harm reduction

Carcinogens

Chemical components

Filter

\begin{abstract}
A B S T R A C T
Background: This study assessed the impact of the diameter and length of different brands on the amount of tobacco in cigarettes and measured the effect of each millimeter length of cigarette filter on the absorption of chemical components in cigarette smoke.

Methods: We selected 11 common brands of domestic and foreign cigarettes, including Bahman-L, Bahman-S, Sater, Montana, Magna, Kent, Marlboro, Winston, Cima, Bistoon, and G1. We measured the length and diameter of each cigarette using a digital caliper with the sensitivity of $0.01 \mathrm{~mm}$ and the amount of tobacco using a digital weighing scale with the sensitivity of $0.0001 \mathrm{gm}$. We assessed the effect of each millimeter length of cigarette filter on the absorption of five known human carcinogens in cigarette smoke, including arsenic, cadmium, chromium, benzene, and formaldehyde.

Results: The volume of cigarettes and the amount of tobacco was much more dependent on the diameter of cigarettes than their length. Only 1-2 mm reduction in the diameter of cigarettes can reduce the amount of tobacco in cigarettes by 20\%-40\%, respectively. Moreover, each incremental increase of $1 \mathrm{~mm}$ of the length of cigarette filter increased the absorption of benzene, arsenic, cadmium, chromium, and formaldehyde by $1.5 \%$, $1.3 \%, 1.9 \%, 2.0 \%$, and $0.7 \%$, respectively.

Conclusion: A minor reduction in the diameter of cigarettes had a substantial impact on the amount of tobacco in each cigarette stick. Furthermore, the length of the cigarette filter had a significant effect on the absorption of the hazardous chemical components of cigarettes.
\end{abstract}

\section{Introduction}

In 2015, more than 1.1 billion people used tobacco. ${ }^{1}$ The prevalence of using tobacco in Iran is about $23.7 \%$ for men and $3.0 \%$ for women. ${ }^{2}$ Tobacco use is a major risk factor for noncommunicable diseases. ${ }^{3-5}$ In 2015, smoking was responsible for one out of ten deaths, killing more than six million people worldwide. ${ }^{6}$ Smoking is responsible for $10 \%$ of all deaths from cardiovascular diseases, $22 \%$ of all deaths from cancer, and $36 \%$ of all deaths from chronic respiratory diseases. ${ }^{7}$

In response to the global tobacco epidemic, the WHO Framework Convention on Tobacco Control (WHO FCTC), the United Nations Tobacco Control Treaty was developed. ${ }^{8}$ WHO FCTC requires all Member States to implement policies designed to reduce both the demand for tobacco products and their supply. These policies include raising prices and taxes on tobacco; banning smoking in public places; use of pictorial health warnings; bans on tobacco advertising; controlling the illicit trade in tobacco products; identifying alternative crops to tobacco farming; preventing sales to and by minors; and collecting and sharing data on tobacco use and prevention efforts. Although there has been significant progress, implementation of the WHO FCTC has been lagging in some areas including controlling the illicit trade, tobacco taxation, the use of liability as a tobacco control measure and promotion of alternative livelihoods for tobacco growers. ${ }^{9-13}$

Besides the aforementioned strategies adopted to reduce tobacco products, the dimensions of cigarettes in terms of the circumference and length can be considered as a harm reduction approach to reduce tobacco consumption. This strategy can reduce the risk of smoking-related diseases not only among smokers but also among ex-smokers. Therefore, this harm reduction approach may have a direct and substantial impact on smoke emissions. Of course, we should confirm that this harm reduction approach is just a hypothesis that has not been tested yet. In other words, it is not clear that by reducing the diameter of cigarettes, smokers will actually use less amount of tobacco or they will change their behaviors by increasing the number of cigarettes to

\footnotetext{
* Corresponding author.

E-mail address: f.gohariensaf94@gmail.com (F. Gohari-Ensaf).
} 
compensate the amount of tobacco they use per day. There is not enough evidence to indicate that slim cigarettes per se are any less harmful than conventional cigarettes with higher circumferences. ${ }^{14}$ Therefore, the actual impact of the diameter reduction on tobacco consumption needs further investigation.

By now, evidence has shown that decreasing cigarette circumference can influence the physical characteristics of the cigarette and hence the nature of the smoke emissions. Decreasing circumference reduces tobacco weight, mass burn rate, puff count and static burn time. In addition, deliveries of individual mainstream and sidestream smoke emissions, including free radical species, in vitro specific mutagenic, cytotoxic and tumorigenic activities tend to decrease as cigarette circumference decreases while formaldehyde increases relative to tar as circumference decreases. ${ }^{14}$ However, further investigations are required to assess the effect of slimmer designs on smokers' behaviors.

It seems possible that a minor modification of the dimensions of cigarettes can reduce mainstream smoke emissions. According to our preliminary assessment, we realized that the diameter and length of cigarettes vary from one brand to another and these characteristics had a substantial impact on the amount of tobacco in cigarettes. However, based on experimental studies is required to realize how much reduction in the diameter and length of cigarettes can have a substantial impact on the amount of tobacco within each cigarette stick. A better understanding of the effect of diameter and length of cigarettes on the amount of tobacco is required to implement effective harm reduction measures and to design effective intervention strategies. In this study, we assessed the impact of diameter and length of 11 types of the most commonly used brands on the amount of tobacco in cigarettes. Furthermore, we measured the effect of each millimeter length of cigarette filter on the absorption of five important toxicants in cigarette smoke.

\section{Methods}

This study was conducted in Hamadan Province, the west of Iran, in 2017. The Vice-chancellor of Research and Technology of Hamadan University of Medical Sciences supported this study and the Research Council of the university approved the protocol (No. 9510075667).

In 2016, a cross-sectional study was conducted on 3480 smokers in six provinces of Iran to assess the status of cigarette marketing in the country. This study evaluated 160 tobacco products marketed in Iran, including 38 (23.8\%) domestic brands and 122 (76.2\%) foreign brands. ${ }^{10}$ Based on the results of this study, we selected 11 most commonly used domestic brands, including Bahman-L (large) and BahmanS (small) (32.9\%), Bistoon (24.8\%), G1 (12.4\%), Cima (6.5\%), and Sater (4.4\%); as well as foreign brands, including Winston $(25.5 \%)$, Kent (18.5\%), Magna (15.6\%), Marlboro (8.1\%), and Montana (5.2\%) (Fig. 1).

Cigarettes with circumferences in the range 14-19 are referred to as super slim, those in the range of 19-21 mm are termed demi slim, those with circumferences of $21-24 \mathrm{~mm}$ are referred to as slim, and cigarettes with circumferences of $24-25 \mathrm{~mm}$ are termed traditional king size. ${ }^{14}$ Based on the circumference of cigarettes, four cigarettes (Sater, Bistoon, G1, and Cima) were supper slim $(14-19 \mathrm{~mm})$; one cigarette was Bahman-S was demi slim (19-21 mm); three cigarettes (Magna, Bahman-L and Winston) were slim (21-24 mm); and three cigarettes (Kent, Marlboro, and Montana) were king-size (24-25 mm).

We measured the length (with and without filter) and the diameter of each cigarette using a digital caliper with the sensitivity of $0.01 \mathrm{~mm}$ (Mitutoyo, CD-8" CSX, made in Japan). Then, we measured the amount of tobacco in each cigarette using a digital weighing scale with the sensitivity of $0.0001 \mathrm{gm}$ (Sartorius, Model Practum, made in Germany). We also calculated the pure volume of each cigarette without a filter by calculating the volume of the cylinder $\left(\mathrm{V}=\pi \mathrm{r}^{2} \mathrm{~h}\right)$, where ' $\mathrm{r}$ ' was the radius and ' $\mathrm{h}$ ' was the pure length of each cigarette.

Tobacco smoke contains over 7000 toxic chemicals, including

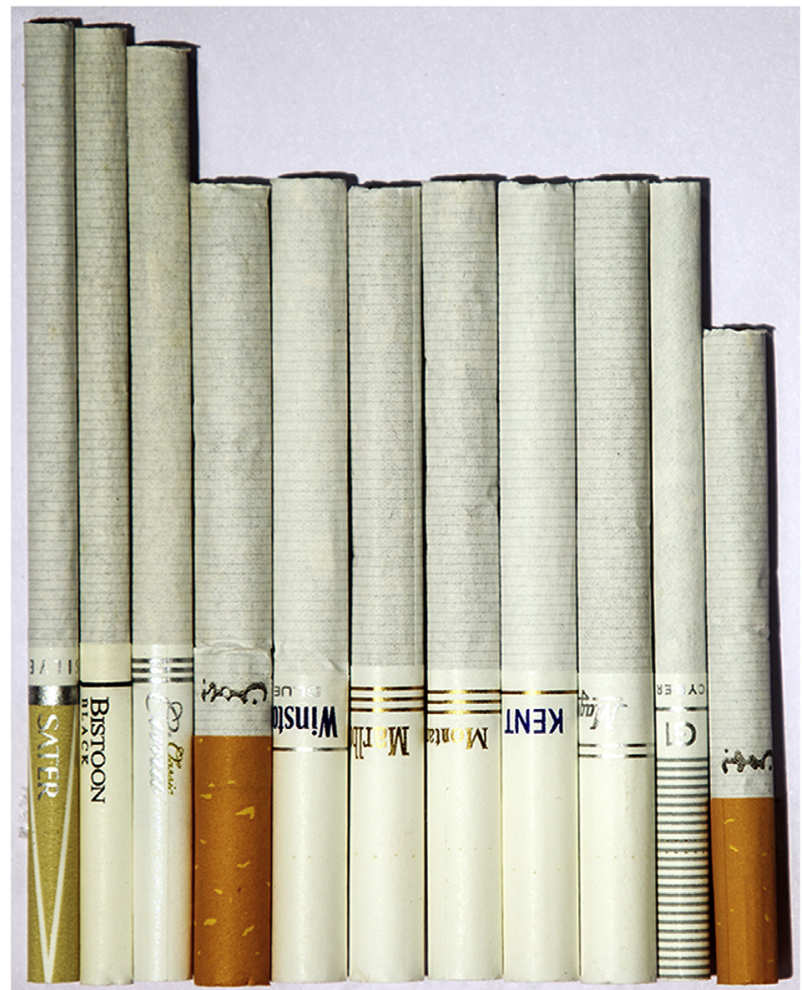

Fig. 1. The appearance of 11 different types of the most commonly used brands of cigarette evaluated in this study.

human carcinogens. ${ }^{15}$ We selected four chemical components in cigarette smoke which were classified Class I (known human carcinogens) and one Class 2A (probable human carcinogens) based on IARC classifications ${ }^{16}$ with the highest cancer risk. ${ }^{17}$ These chemical components included arsenic, cadmium, chromium, benzene, and formaldehyde. In order to assess the effect of cigarette filter on the absorption of chemical components, we measured the chemical components twice, once with the whole filter and once without a filter. In order to align the study conditions for all types of cigarettes, and to remove the effect of filter vents, we wrapped the cigarette filters in a silicon probe.

We measured heavy metals based on NIOSH Manual of Analytical Methods (NMAM), ${ }^{18} 4$ th edition, including 'As': METHOD 7900 for arsenic, 'Cd': METHOD 7048 for cadmium, and 'Cr': METHOD 7024 for chromium. We used a membrane cellulose cell filter with a pore of 0.8 mic and a diameter of $37 \mathrm{~mm}$ to sample the heavy metals in the cigarette smoke. We put the sampling filter into a filter cassette and connected the filter cassette to an individual sampling pump with a flow rate of $215 \mathrm{ml} / \mathrm{min}$. Then, we burned the cigarette and simultaneously started and continued pumping until the end of the cigarette. We carried out this process twice, with and without a filter, for each of the 11 brands, in order to evaluate the effect of cigarette filter on the absorption of heavy metals. We analyzed heavy metal samples by using PerkinElmer AAnalyst 700 Furnace Atomic Absorption Spectrometer.

We measured benzene by using a solid Sorbent tube based on NMAM, Hydrocarbons, Aromatic, METHOD 1501. We used activated charcoal from coconut skin. We put $100 \mathrm{mg}$ of the activated charcoal in the front part and $50 \mathrm{mg}$ in the rear part of a pipe with a length of $7 \mathrm{~cm}$ and an inner diameter of $4 \mathrm{~mm}$ and an outer diameter of $6 \mathrm{~mm}$. We connected the cigarette to the front part of the pipe and the individual sampling pump to the rear part of the pipe with a flow rate of $170 \mathrm{ml} /$ min. Then, we burned the cigarette and simultaneously started and continued pumping until the end of the cigarette. We carried out this process twice, with and without a filter, for each of the 11 brands, in 
order to evaluate the effect of cigarette filter on the absorption of benzene. We analyzed samples of benzene by using Varian CP-3800 GCMS.

We measured formaldehyde based on NMAM, METHOD 3500. For this purpose, we used three sequential sampling impingers, including $15 \mathrm{mg}$ sodium bisulfite $1 \%$. We connected the cigarette to the first impinger through a Teflon filter, in order to prevent particle pollutants from entering the circuit. We connected the pump, with a flow rate of $190 \mathrm{ml} / \mathrm{min}$, to the third impinger through a trap, in order to prevent gases and vapors entering the pump. Then, we burned the cigarette and simultaneously started and continued pumping until the end of the cigarette. We carried out this process twice, with and without a filter, for each of the 11 brands, in order to evaluate the effect of cigarette filter on the absorption of formaldehyde. We analyzed samples of formaldehyde by using PerkinElmer Lambda 950 Spectrophotometer.

The independent $t$-test was used to compare means. The linear regression was used to assess the effect of $1 \mathrm{~mm}$ reduction in the diameter cigarettes on the amount of tobacco and the relationship between the length of the cigarette filter and the absorption of chemical components in cigarette smoke. All statistical analyses were performed at a significance level of 0.05 using Stata software, version 14 (StataCorp). Microsoft Excel, version 2016, was used for drawing the figure.

\section{Results}

In this study, 11 different types of the most commonly used cigarettes were evaluated. The total length of cigarettes, the length of filters, the pure length of cigarettes, the diameter and volume of cigarettes and the amount of tobacco in each cigarette are reported in Table 1. The amount of tobacco in cigarettes varied significantly from $0.2960 \mathrm{gm}$ to $0.6686 \mathrm{gm}$ depending on the length and diameter of the cigarettes.

We compared the characteristics of cigarettes in Fig. 2. As shown in this figure, the volume of the cigarettes decreases as the diameter decreases, even though the length of the cigarette may even increase. In other words, the volume of the cigarettes and hence the amount of tobacco in cigarettes is much more dependent on the diameter of the cigarettes than to their length.

The effect of reduction in the diameter of cigarettes on the amount of tobacco based on the multiple linear regression is shown in Table 2. According to results of this table, $1 \mathrm{~mm}$ reduction in the diameter of cigarettes will reduce the amount of tobacco by about $20 \%$ and $2 \mathrm{~mm}$ reduction in the diameter of cigarettes will reduce the amount of tobacco by about $40 \%$ and so on.

The effect of cigarette filter on the reduction of chemical components in cigarette smoke is given in Table 3. On average, cigarette filter can reduce the amount of benzene by $45 \%$, arsenic by $38 \%$, cadmium by $49 \%$, chromium by $57 \%$, and formaldehyde by $57 \%$. Each incremental increase of $1 \mathrm{~mm}$ of the length of cigarette filter can increase, on average, the absorption of benzene by $1.5 \%$, arsenic by $1.3 \%$, cadmium by $1.9 \%$, chromium by $2.0 \%$ and formaldehyde by $0.7 \%$.

\section{Discussion}

On the basis of our findings, the amount of tobacco in the cigarette is strongly dependent on the diameter of cigarettes. Therefore, only 1-2 mm reduction in the diameter of cigarettes can reduce the amount of tobacco in cigarettes by $20 \%-40 \%$, respectively. Such a minor reduction in the diameter of cigarettes has a great impact on the amount of tobacco consumption. Heavy smokers usually use one or two cigarette packets or even more every day. They are more dependent on the number of cigarettes than the amount of tobacco they use. In such a situation, if the diameter of the cigarette is reduced by 1 or $2 \mathrm{~mm}$, the amount of tobacco consumption may reduce by $20-40 \%$ without smokers realize it.

Currently, super slim cigarettes are becoming popular worldwide. ${ }^{14}$ The introduction of the super slim cigarettes, which were originally tailored specifically for the female consumers, are of concern because slimmer designs are interpreted by consumers to produce lower toxic emissions and are perceived to be less harmful. ${ }^{19,20}$ Kant et al. conducted a study and compared the harmful particulate matter concentrations emitted by slim-size cigarettes versus king-size cigarettes. They concluded that slim-size cigarettes potentially more harmful for passive smokers than king-size cigarettes. ${ }^{21}$ Siu et al. showed that the Canadian super slim cigarette resulted in lower yields of carbon monoxide, the carbonyls, volatiles and the aromatic amines in the mainstream smoke emissions. However, it significantly increased some toxicant levels in the mainstream smoke emissions, including formaldehyde, ammonia and the phenols. ${ }^{22}$ Epidemiological studies have shown that decreasing cigarette circumference can influence the physical properties of the cigarette and hence the nature of the smoke produced. ${ }^{14}$

We indicated that each incremental increase of $1 \mathrm{~mm}$ of the length of the cigarette filter can reduce the inhalation of chemical components in cigarette smoke by about $2 \%$. Therefore, $5 \mathrm{~mm}$ increase in the length of cigarettes can have a significant impact on the reduction of toxic chemical components inhaled during cigarette smoking. This strategy has mutual benefits. A minor increase in the length of cigarette filter not only reduces the inhalation of carcinogenic components in cigarette smoke but also reduces the amount of tobacco in cigarettes if the length of cigarettes is considered constant.

Evidence showed that smoke emissions are affected by several factors. King et al. investigated the mainstream smoke emissions of 15 Australian and 21 Canadian brands and showed that country and manufacturer variables were the strongest predictors of intensive condition adjusted emissions for 8 of the 13 hazardous agents. ${ }^{23}$ Djordjevic et al. carried out a study to obtain more realistic estimates of exposure to carcinogenic components of cigarette smoke. They used a pressure transducer system and assessed puffing characteristics for 133 low- and medium-yield cigarette smokers. They indicated that compared with the FTC protocol values, smokers of low- and medium-yield brands took larger puffs at shorter intervals and drew larger total smoke volumes. They concluded that the FTC protocol underestimates doses of nicotine

Table 1

The characteristics of 11 types of the most commonly used cigarettes enrolled in this study in descending order of circumference of cigarettes.

\begin{tabular}{|c|c|c|c|c|c|c|c|}
\hline Cigarettes & Total length (mm) & Filter length (mm) & Pure length (mm) & Diameter (mm) & Circumference (mm) & Pure volume $\left(\mathrm{mm}^{3}\right)$ & Tobacco (gm) \\
\hline Montana & 82.98 & 27.10 & 55.88 & 7.78 & 24.43 & 2655.13 & 0.5885 \\
\hline Kent & 82.22 & 27.10 & 55.12 & 7.68 & 24.12 & 2552.12 & 0.5459 \\
\hline Marlboro & 83.25 & 27.10 & 56.15 & 7.68 & 24.12 & 2599.81 & 0.5205 \\
\hline Winston & 83.68 & 27.10 & 56.58 & 7.57 & 23.77 & 2545.21 & 0.4848 \\
\hline Bahman-L & 83.24 & 20.74 & 62.50 & 7.55 & 23.71 & 2796.69 & 0.6686 \\
\hline Magna & 83.40 & 27.70 & 55.70 & 7.48 & 23.49 & 2446.40 & 0.5485 \\
\hline Bahman-S & 68.50 & 11.03 & 57.47 & 6.14 & 19.28 & 1700.78 & 0.4735 \\
\hline Cima & 96.81 & 30.56 & 66.25 & 5.83 & 18.31 & 1767.64 & 0.4332 \\
\hline G1 & 82.48 & 27.25 & 55.23 & 5.37 & 16.86 & 1250.24 & 0.2960 \\
\hline Bistoon & 98.97 & 30.43 & 68.54 & 5.22 & 16.39 & 1466.07 & 0.3322 \\
\hline Sater & 99.57 & 29.84 & 69.73 & 5.20 & 16.33 & 1480.12 & 0.3824 \\
\hline
\end{tabular}




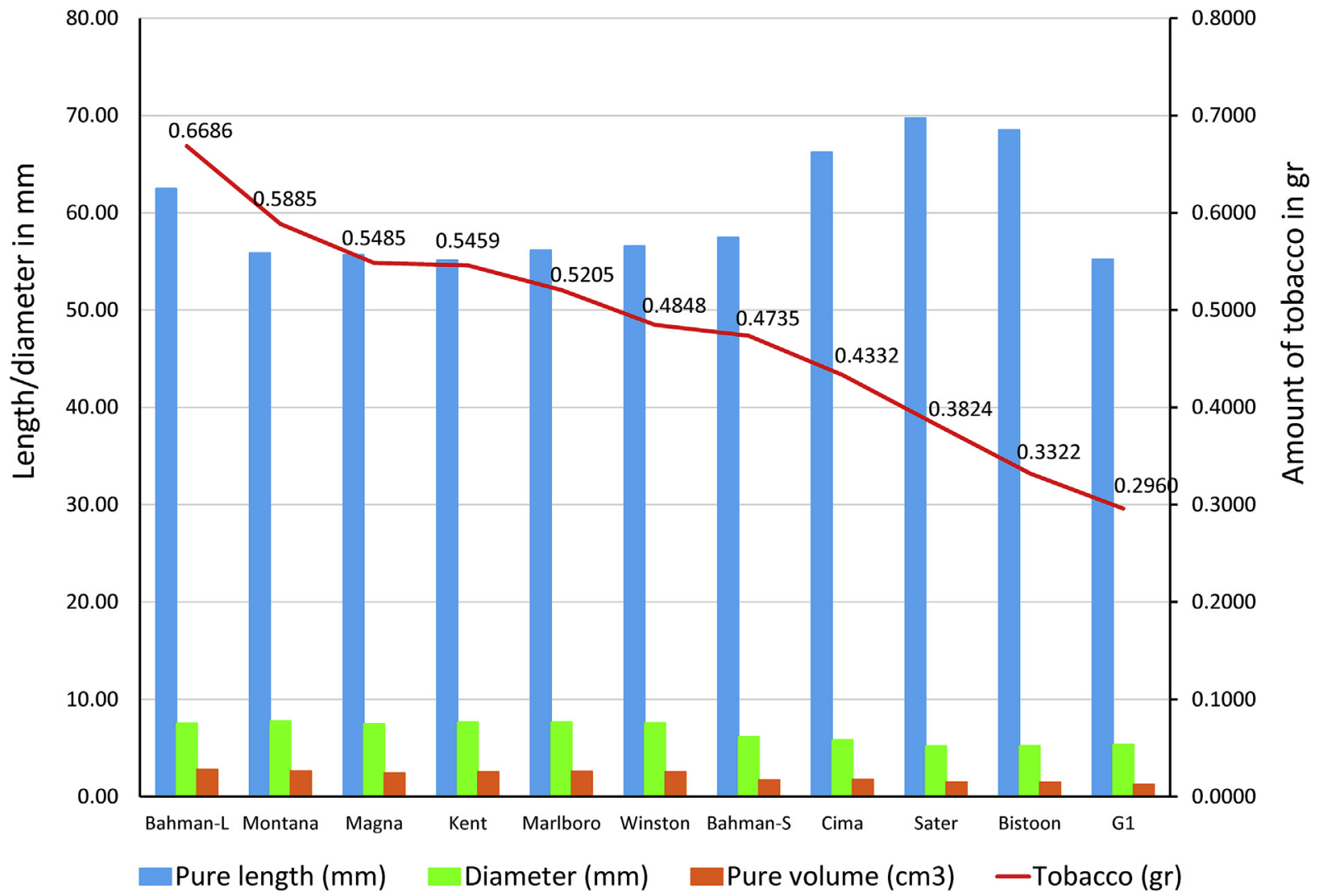

Fig. 2. Comparison of the characteristics of 11 different types of cigarettes evaluated in this study indicating the decreasing trend of tobacco gradients in line with decrease in diameter of cigarettes.

and carcinogen received by smokers and overestimates the proportional benefit of low-yield cigarettes. ${ }^{24}$

Several studies evaluated the effect of the filters on cigarette smoking behaviors. Unexpectedly, the epidemiological data are against cigarette filter. Kozlowski et al. showed that filters misleadingly make cigarettes taste lighter and milder, and, therefore, they may appear more delicious and less harmful to smokers. In addition, the filter may promote smokers to compensate for a lower dose of nicotine by taking larger puffs. Furthermore, behavioral filter vent blocking with lips or fingers is an additional contributor to compensatory smoking. ${ }^{25}$ Current evidence has shown that tiny ventilation holes in cigarette filters increase the risk for adenocarcinoma of the lung. The reason is that the ventilation holes in the filters change the smokers' inhalation behavior and cause them to inhale more vigorously drawing carcinogens more deeply into the lungs. ${ }^{26,27}$ Even though filtered brands now make up over $90 \%$ of the entire cigarette market, epidemiological data gathered during the past four decades continue to support the conclusion that filtered cigarettes have not done anything to mitigate the health hazards of smoking. ${ }^{28}$ In 2010, a population-based survey in the USA and Japan indicated that the shift in tobacco from nonfilter to filter cigarettes appears to have merely altered the incidence of the most frequent type of lung cancer, from squamous cell carcinoma to adenocarcinoma. ${ }^{29}$

The main limitation of this study was that it was actually impossible to measure the impact of every $1 \mathrm{~mm}$ incremental increase in cigarette filter on the absorption of chemical components in cigarette smoke.

Table 2

The effect of reduction in the diameter of cigarettes on the amount of tobacco based on the multiple linear regression adjusted for the length of the cigarette.

\begin{tabular}{llllll}
\hline Variables & Coefficient & $\mathrm{SD}$ & $\mathrm{t}$ & $P$ value & $95 \% \mathrm{CI}$ \\
\hline Pure length & 0.007158 & 0.003672 & 1.95 & 0.087 & -0.001309 \\
Diameter & 0.112044 & 0.018840 & 5.95 & 0.000 & 0.068600 \\
Constant & -0.698114 & 0.317833 & -2.20 & 0.059 & -1.431037 \\
\hline
\end{tabular}

Equation: $\mathrm{y}=-0.698114+(0.112044 \times$ Diameter $)+(0.007158 \times$ Pure length $)$.

Example.

A cigarette with a diameter of $7 \mathrm{~mm}$ and a length of $70 \mathrm{~mm}$ contain.

Tobacco $=-0.698114+(0.112044 \times 7)+(0.007158 \times 70)=0.5872492 \mathrm{gr}$.

A cigarette with a diameter of $6 \mathrm{~mm}$ and a length of $70 \mathrm{~mm}$ contain.

Tobacco $=-0.698114+(0.112044 \times 6)+(0.007158 \times 70)=0.4752049 \mathrm{gr}$.

A cigarette with a diameter of $5 \mathrm{~mm}$ and a length of $70 \mathrm{~mm}$ contain.

Tobacco $=-0.698114+(0.112044 \times 5)+(0.007158 \times 70)=0.3631606$ gr.

If the diameter of a cigarette reduces from $7 \mathrm{~mm}$ to $6 \mathrm{~mm}$ ( $1 \mathrm{~mm}$ reduction), the amount of tobacco will reduce from $0.5872492 \mathrm{gr}$ to $0.4752049 \mathrm{gr}$, which is about $20 \%$ reduction in the amount of tobacco.

If the diameter of a cigarette reduces from $7 \mathrm{~mm}$ to $5 \mathrm{~mm}$ ( $2 \mathrm{~mm}$ reduction), the amount of tobacco will reduce from $0.5872492 \mathrm{gr}$ to 0.3631606 gr, which is about $40 \%$ reduction in the amount of tobacco. 
Table 3

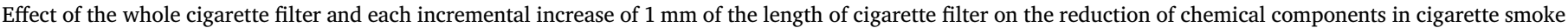
based on linear regression model.

\begin{tabular}{|c|c|c|c|c|c|c|c|}
\hline \multirow[t]{2}{*}{ Chemical components in cigarette smoke } & \multicolumn{2}{|c|}{ Cigarette filter } & \multicolumn{2}{|c|}{ Observed reduction by the whole filter } & \multirow[t]{2}{*}{$t$-test $\mathrm{P}$ value } & \multicolumn{2}{|c|}{ Estimated reduction by every $1 \mathrm{~mm}$ of filter } \\
\hline & Without & With & Absolute & Percent & & Absolute & Percent \\
\hline Benzene $(\mu \mathrm{g} / \mathrm{cig})$ & 80.45 & 44.15 & 36.30 & $45 \%$ & 0.003 & $1.24^{\mathrm{a}}$ & $1.5 \%$ \\
\hline Arsenic $(\mu \mathrm{g} / \mathrm{cig})$ & 20.38 & 12.73 & 7.65 & $38 \%$ & 0.001 & $0.26^{\mathrm{b}}$ & $1.3 \%$ \\
\hline Cadmium ( $\mu \mathrm{g} / \mathrm{cig})$ & 11.41 & 5.80 & 5.61 & $49 \%$ & 0.001 & $0.22^{\mathrm{c}}$ & $1.9 \%$ \\
\hline Chromium ( $\mu \mathrm{g} / \mathrm{cig})$ & 11.24 & 4.83 & 6.41 & $57 \%$ & 0.001 & $0.22^{\mathrm{d}}$ & $2.0 \%$ \\
\hline Formaldehyde ( $\mu \mathrm{g} / \mathrm{cig})$ & 92.32 & 78.68 & 13.64 & $15 \%$ & 0.303 & $0.61^{\mathrm{e}}$ & $0.7 \%$ \\
\hline
\end{tabular}

a $y=\beta_{0}+\beta_{1} x_{1}=78.35-1.24 x_{1}$, where $x_{1}$ is the length of cigarette filter in $\mathrm{mm}$.

b $y=\beta_{0}+\beta_{1} x_{1}=19.98-0.26 x_{1}$, where $x_{1}$ is the length of cigarette filter in mm.

c $y=\beta_{0}+\beta_{1} x_{1}=11.41-0.22 x_{1}$, where $x_{1}$ is the length of cigarette filter in $\mathrm{mm}$.

d $y=\beta_{0}+\beta_{1} x_{1}=10.92-0.22 x_{1}$, where $x_{1}$ is the length of cigarette filter in mm.

e $y=\beta_{0}+\beta_{1} x_{1}=93.39-0.61 x_{1}$, where $x_{1}$ is the length of cigarette filter in $\mathrm{mm}$.

Therefore, we calculated it mathematically. This might introduce information bias in our results. In addition, the type and quality of the materials used in filters may influence the absorption rate. However, we did not evaluate the quality of the filters and their impact on the inhalation of toxic chemical components of cigarette smoke.

\section{Conclusion}

Based on our evidence, the length of cigarettes filter had a significant effect on the absorption of the hazardous chemical components of cigarettes. Furthermore, a minor reduction in the diameter of the cigarette had a substantial impact on the amount of tobacco in each cigarette stick, which is very important in risk reduction programs. In other words, a minor reduction in the diameter of the cigarette will have a tremendous effect on the reduction of tobacco consumption and its health-related consequences. However, the actual impact of the diameter reduction on tobacco consumption needs further investigation.

\section{Ethics approval and consent to participate}

This study was conducted in laboratory and did not involve human participants, human data, human tissue, or animal.

\section{Sources of funding}

The Vice-Chancellor of Research and Technology, Hamadan University of Medical Sciences funded this study (No. 9510075667).

\section{Author contributions}

JP contributed to study conception and design, analysis and interpretation of data, and drafting the manuscript. MJA contributed to study design, acquisition of data and critical revision. YM contributed to the study design and critical revision. FGE contributed to study design, acquisition of data, analysis, and interpretation of data, and critical revision.

\section{Declaration of competing interest}

The authors have no conflict of interest to declare.

\section{Acknowledgments}

This was part of the MSc thesis in Epidemiology. We would like to appreciate the Vice-Chancellor for Research and Technology of the Hamadan University of Medical Sciences for approval of this work.

\section{References}

1. World Health Organization. Monitoring Health for the SDGs Sustinable Development Goals. Geneva: WHO; 2016

2. Nemati S, Rafei A, Freedman ND, Fotouhi A, Asgary F, Zendehdel K. Cigarette and water-pipe use in Iran: geographical distribution and time trends among the adult population; a pooled analysis of national steps surveys, 2006-2009. Arch Iran Med. 2017;20:295-301.

3. World Health Organization. Global Atlas on Cardiovascular Disease Prevention and Control. Geneva: WHO; 2011.

4. Poorolajal J, Bahrami M, Karami M, Hooshmand E. Effect of smoking on multiple sclerosis: a meta-analysis. J Public Health (Oxf). 2017;39:312-320.

5. Poorolajal J, Darvishi N. Smoking and suicide: a meta-analysis. PLoS One. 2016;11:e0156348.

6. GBD 2015 Tobacco Collaborators. Smoking prevalence and attributable disease burden in 195 countries and territories, 1990-2015: a systematic analysis from the global burden of disease study. Lancet. 2017;389:1861-1862.

7. world Health Organization. WHO Global Report: Mortality Attributable to Tobacco. Geneva: WHO; 2012

8. World Health Organization. WHO Framework Convention on Tobacco Control. Geneva: WHO; 2003.

9. World Health Organization. Global Progress Report on Implementation of the WHO Framework Convention on Tobacco Control Geneva: WHO; 20162016.

10. Poorolajal J, Mohammadi Y, Mahmoodi A. Challenges of tobacco control program in Iran. Arch Iran Med. 2017;20:229-234.

11. Ross H, Husain MJ, Kostova D, et al. Approaches for controlling illicit tobacco tradenine countries and the European Union. MMWR Morb Mortal Wkly Rep. 2015;64:547-550.

12. Joossens L, Merriman D, Ross H, Raw M. The impact of eliminating the global illicit cigarette trade on health and revenue. Addiction (Abingdon, England). 2010;105:1640-1649.

13. Samji H, Wardman D. First Nations Communities and Tobacco Taxation: A Commentary. American Indian and Alaska Native Mental Health Research (Online). vol. 16. 2009; 2009:1-10.

14. McAdam K, Eldridge A, Fearon IM, et al. Influence of cigarette circumference on smoke chemistry, biological activity, and smoking behaviour. Regul Toxicol Pharmacol : RTP. 2016;82:111-126.

15. World Health Organization. World No Tobacco Day 2017: Beating Tobacco for Health, Prosperity, the Environment and National Development. Geneva: WHO; 2017. [cited 1 June 2017]; Available from. http://www.who.int/mediacentre/news/releases/ 2017/no-tobacco-day/en/.

16. International Agency for Research on Cancer (IARC). WHO/IARC classification of tumours. . [cited 4 June 2017]; Available from. http://publications.iarc.fr/BookAnd-Report-Series/Who-Iarc-Classification-Of-Tumours; 2017.

17. Fowles J, Bates M, Noiton D. The Chemical Constituents in Cigarettes and Cigarette Smoke: Priorities for Harm Reduction. New Zealand: Institute of Environmental Science and Research Limited; 2000.

18. The National Institute for Occupational Safety and Health. NIOSH Manual of Analytical Methods. Atlanta: CDC; 2017. [updated August 23, 2016; cited 3 Jun, 2017]; Available from. https://www.cdc.gov/niosh/nmam/.

19. Mutti S, Hammond D, Borland R, Cummings MK, O'Connor RJ, Fong GT. Beyond light and mild: cigarette brand descriptors and perceptions of risk in the International Tobacco Control (ITC) Four Country Survey. Addiction (Abingdon, England). 2011;106:1166-1175.

20. Hammond D, Doxey J, Daniel S, Bansal-Travers M. Impact of female-oriented cigarette packaging in the United States. Nicotine Tob Res : Off J Soc Res Nicotine Tob. 2011;13:579-588.

21. Kant N, Muller R, Braun M, Gerber A, Groneberg D. Particulate matter in secondhand smoke emitted from different cigarette sizes and types of the brand vogue mainly smoked by women. Int J Environ Res Public Health. 2016;13.

22. Siu M, Mladjenovic N, Soo E. The analysis of mainstream smoke emissions of Canadian 'super slim' cigarettes. Tob Control. 2013;22:e10.

23. King B, Borland R, Fowles J. Mainstream smoke emissions of Australian and 
Canadian cigarettes. Nicotine \& tobacco research. Nicotine Tob Res : Off J Soc Res Nicotine Tob. 2007;9:835-844.

24. Djordjevic MV, Stellman SD, Zang E. Doses of nicotine and lung carcinogens delivered to cigarette smokers. J Natl Cancer Inst. 2000;92:106-111.

25. Kozlowski LT, O'Connor RJ. Cigarette filter ventilation is a defective design because of misleading taste, bigger puffs, and blocked vents. Tob Control. 2002;11(Suppl 1):I40-I50.

26. Samet JM, Aladadyan L. Should the FDA ban cigarette filter ventilation? J Natl Cancer Inst. 2017;109.
27. Song MA, Benowitz NL, Berman M, et al. Cigarette filter ventilation and its relationship to increasing rates of lung adenocarcinoma. J Natl Cancer Inst. 2017;109. 28. Harris B. The intractable cigarette 'filter problem. Tob Control. 2011;20(Suppl 1):i10-i16.

29. Ito $\mathrm{H}$, Matsuo $\mathrm{K}$, Tanaka $\mathrm{H}$, et al. Nonfilter and filter cigarette consumption and the incidence of lung cancer by histological type in Japan and the United States: analysis of 30-year data from population-based cancer registries. Int $J$ Cancer. 2011;128:1918-1928. 